\title{
The Use of a Cell Filter for State Estimation in Closed-Loop NMPC of Low Dimensional Systems
}

Sridhar Ungarala

Cleveland State University

Keyu Li

Cleveland State University

Follow this and additional works at: https://engagedscholarship.csuohio.edu/encbe_facpub

Part of the Process Control and Systems Commons

How does access to this work benefit you? Let us know!

Publisher's Statement

NOTICE: this is the author's version of a work that was accepted for publication in Journal of

Process Control. Changes resulting from the publishing process, such as peer review, editing, corrections, structural formatting, and other quality control mechanisms may not be reflected in this document. Changes may have been made to this work since it was submitted for publication. A definitive version was subsequently published in Journal of Process Control, 19, 3, (March 2009) DOI 10.1016/j.jprocont.2008.04.020

\section{Original Citation}

Ungarala, S., , \& Li, K. (2009). The use of a cell filter for state estimation in closed-loop NMPC of low dimensional systems. Journal of Process Control, 19(3), 550-556. doi:10.1016/j.jprocont.2008.04.020

\section{Repository Citation}

Ungarala, Sridhar and Li, Keyu, "The Use of a Cell Filter for State Estimation in Closed-Loop NMPC of Low Dimensional Systems" (2009). Chemical \& Biomedical Engineering Faculty Publications. 50.

https://engagedscholarship.csuohio.edu/encbe_facpub/50

This Article is brought to you for free and open access by the Chemical \& Biomedical Engineering Department at EngagedScholarship@CSU. It has been accepted for inclusion in Chemical \& Biomedical Engineering Faculty Publications by an authorized administrator of EngagedScholarship@CSU. For more information, please contact library.es@csuohio.edu. 


\title{
The use of a cell filter for state estimation in closed-loop NMPC of low dimensional systems
}

\author{
Sridhar Ungarala *, Keyu Li \\ Department of Chemical and Biomedical Engineering. Cleveland State University, 2121 Euclid Avenue, SH 455, Cleveland, OH 44115, USA
}

\section{Introduction}

General model predictive control (MPC) solves an open-loop optimal control problem in a finite horizon based on the prediction of future dynamics originating from the current state of the process. Only the first control action is implemented and a new control profile is obtained at the next sampling time based on updated state information. However, directly measuring the entire state vector may not be economical or even feasible. Using available measurements and dynamic models, an estimation algorithm is used to reconstruct the state vector, which is then used as feedback to the MPC regulator. Since the quality of prediction and the optimality of control profile are dependent on the initial condition fed back, both the accuracy and speed of estimation are critical for the performance of the regulator.

The use of nonlinear models in estimation and predictive control is gaining importance in the face of performance limitations faced by linear MPC and Kalman filter when applied to nonlinear processes commonly encountered in chemical and biological systems. There is a small but growing list of industrial MPC applications based on nonlinear models [1]. The major challenge is the execution of nonlinear estimator-regulator solution in real-time, which can have a strong influence on the stabilizing properties of the regulator [2]. Several computationally feasible approaches for the regulator problem have been demonstrated recently such as successive quadratic programming [3], off-line preparation and feedback [4] and in situ adaptive tabulation [5].

The state estimation task may be posed as a general recursive Bayesian inference problem. For linear dynamic processes, the mean vectors and covariance matrices are sufficient summary statistics to represent the variables. Hence, iterative relationships for the conditional mean and covariance of the states track the evolution of the conditional probability density function (pdf) of the states. However, process nonlinearity can render the conditional pdf into a time-varying multimodal non-Gaussian, which is distorted, spread and translated at each time instance. It is widely recognized that it is generally not possible to compute the infinite dimensional conditional pdf for real-time applications in a recursive manner.

Originally the extended Kalman filter (EKF) was used as a state estimator in conjunction with nonlinear MPC and it is still popular in industrial applications due to its simplicity [6]. More recently, moving horizon estimation (MHE) has gained attention due to its superior estimation properties [3]. The ability to impose constraints has been the most appealing feature of state estimation by moving horizon formulation. Several authors derived a probabilistic formulation and interpreted the MHE as a general Bayesian estimator [7-9]. Closed-loop nonlinear MPC based on MHE is a topic of recent investigations [3]. The presence of two nested nonlinear programs is a challenge for practical applications. In some cases the optimization in MHE may have larger number of degrees of freedom than the MPC regulator. Advances in nonlinear programming may alleviate the computational demands of MHE, while real-time implementations remain a challenge. 
During recent years there has been a revived interest in solving recursive Bayesian inference problems without simplifying assumptions about nonlinearity or non-Gaussianity. Density based methods such as sequential Monte Carlo (SMC) filter has been shown to be more accurate and faster than optimization based methods [10,11]. Similar to SMC the cell filter (CF) is based on Monte Carlo integration but relies on aggregate Markov chains developed offline, which reduces the online computational burden [12]. Extension of both SMC and CF to constrained systems are also reported [13,14]. So far SMC and CF approaches have been demonstrated in open-loop processes only. The main objective of this paper is to demonstrate the use of the cell filter for closed-loop nonlinear MPC regulation, where an MHE may be avoided. Simulation examples dealing with a nonlinear CSTR and an agricultural pest control problem are discussed.

\section{Closed-loop regulation}

The nonlinear process under consideration is described by a stochastic vector difference equation

$x_{k}=f\left(x_{k-1}, u_{k-1}, w_{k-1}\right), \psi$

where $x_{k}$ is the state vector, $u_{k}$ is the control vector and $w_{k}$ is a vector of uncorrelated random variables distributed according to a known probability density function $p_{w}\left(w_{k}\right)$, which is generally a non-Gaussian pdf. In practice $f$ may be realized via numerical integration of ordinary differential equations (ODEs) over a sampling interval $\Delta t$. During the sampling interval the inputs $u_{k-1}$ and $w_{k-1}$ are considered constant to generate a portion of the state trajectory originating at $x_{k-1}$ and terminating at $x_{k}$.

The state of the process is often not observable directly or completely. It is assumed that the available process measurement vector $y_{k}$ is related to the states through a nonlinear function

$y_{k}=h\left(x_{k}, v_{k}\right), \psi$

where $v_{k}$ denotes uncorrelated random measurement errors distributed according to a known pdf $p_{v}\left(v_{k}\right)$, which is in general a nonGaussian pdf.

Given the knowledge of the current state of the process, say $\hat{x}_{k}$ the regulation task is posed as an open loop optimal control problem inside a finite time horizon extending into the future. A model predictive controller determines the control signal profile over the control horizon $H_{\mathrm{c}}$ based on the prediction of future dynamic behavior over a prediction horizon $H_{\mathrm{p}} \geqslant H_{\mathrm{c}}$, while optimizing a performance criterion. The optimization is posed as follows

$\min _{u_{k, \ldots, k+H_{c}-1}} J=\Gamma\left(x_{k+H_{c}}\right)+\stackrel{k+H_{c}-1}{\sum_{j=k}}\left(\Phi\left(x_{j}, u_{j}\right), \psi\right.$

subject to a deterministic predictive model and constraints:

$x_{k}=f\left(x_{k-1}, u_{k-1}, E\left\{w_{k-1}\right\}\right), \psi$

$x_{k} \in \mathbb{X}, \psi$ and $u_{k} \in \mathbb{U}, \psi$

where Eq. (1) is used for prediction with the stochastic variable $w_{k-1}$ assumed to be constant at its mean value. The sets $\mathbb{X}$ and $\mathbb{U}$ are often considered as boxed polyhedral convex sets.

The performance criterion function $\Phi$, known as the stage cost function, is most often taken as a measure of the deviation of the state variables and the control signal from their respective target values $x^{t}$ and $u^{t}$. The target values are the steady states of the model that correspond to the setpoint value for the output $y^{s}$.The following quadratic form for the performance criterion is frequently used

$$
\begin{aligned}
\Phi\left(x_{k}, u_{k}\right)= & \left(x_{k}-x^{t}\right)^{\mathrm{T}} Q_{c}\left(x_{k}-x^{t}\right)+\left(u_{k}-u^{t}\right)^{\mathrm{T}} R_{c}\left(u_{k}-u^{t}\right) \\
& +\left(u_{k}-u_{k-1}\right)^{\mathrm{T}} S_{\mathrm{c}}\left(u_{k}-u_{k-1}\right), \psi
\end{aligned}
$$

where $Q_{c}, R_{c}$ and $S_{c}$ are symmetric, positive definite weighting matrices. The last term in the above equation represents penalty imposed on changes in control action. The terminal cost function $\Gamma$ is defined as

$\Gamma\left(x_{k+H_{\mathrm{c}}}\right)=x_{k+H_{\mathrm{c}}}^{\mathrm{T}} T_{\mathrm{c}} x_{k+H_{\mathrm{c}}}, \psi$

where the weighting matrix $T_{\mathrm{c}}$ is obtained from the linear quadratic control solution of the linearized system [15].

If one knows the current state $x_{k}$ perfectly and there are no plant-model mismatch or unknown external disturbances, the open loop optimal control profile may be solved for infinite horizons for some cases. The optimized control may then be executed at $k=0$ for all future $k>0$. In general both the initial condition and the predictive model are not known exactly. As a result the expected dynamic behavior inside $H_{\mathrm{c}}$ due to the implementation of the optimized control signal $u_{k, \ldots, k+H_{c}-1}$ will differ from the true state of the process.

When information is available in the form of new state estimates from measurements, disturbance estimates or updated models, a new control profile must be determined by incorporating the new information as feedback. In practice, only the first control value $u_{k}$ is executed as a constant input until the next state estimate is available from the measurements at $k+1$, at which point the horizons are moved forward and the regulation problem is solved once again. The purpose of the state estimation algorithm is to reconstruct both the measured and unmeasured states from noisy measurements to provide an approximate initial condition for the regulation problem. Hence the accuracy and computational efficiency of the estimator are critical for the performance of the regulator.

It is desirable to estimate the temporal evolution of the states in some optimal sense. Since the state vector is a random variable, the complete statistical information about the state is embodied in its probability density conditioned on measurements $p\left(x_{k} \mid y_{k}\right)$. Hence, the state estimator must construct the evolution of the conditional density from which optimal inferences may be drawn as state estimates. The general formulation of the state estimator is posed as the well known recursive Bayesian update problem [16]

$p\left(x_{k} \mid y_{k}\right)=\frac{1}{p\left(y_{k}\right)} p\left(y_{k} \mid x_{k}\right) f p\left(x_{k} \mid x_{k-1}\right) p\left(x_{k-1} \mid y_{k-1}\right) \mathrm{d} x_{k-1}, \psi$

where $p\left(x_{k-1} \mid y_{k-1}\right)$ and $p\left(x_{k} \mid y_{k}\right)$ are the conditional a posteriori densities at time instances $k-1$ and $k$ respectively. The integral term is the $a$ priori density where the state transition probability density is obtained as

$p\left(x_{k} \mid x_{k-1}\right)=p_{w}\left(f^{-1}\left(x_{k}, x_{k-1}, u_{k-1}\right)\right) \mid\left\|\frac{\partial f^{-1}}{\partial x_{k}}\right\|(\psi$

where for fixed $x_{k-1}$ and $u_{k-1}$, the invers of $f\left(x_{k-1}, u_{k-1}, w_{k-1}\right)$ is $f^{-1}$. If the dimension $n$ of the state vector is equal to the dimension $m$ of the random noise vector, the model can be solved for $w_{k-1}$ and $f^{-1}$ is an $n$-dimensional vector function. In case $m<n$ the state vector is partitioned into $x_{k}^{\mathrm{T}}=\left[x_{k}^{(m) \mathrm{T}}, x_{k}^{(n-m) \mathrm{T}}\right]$ and the state transition pdf is

$p\left(x_{k} \mid x_{k-1}\right)=p\left(x_{k}^{(n-m)} \mid x_{k}^{(m)}\right) p\left(x_{k}^{(m)} \mid x_{k-1}\right), \psi$

where $p\left(x_{k}^{(m)} \mid x_{k-1}\right) \stackrel{\text { is }}{ }$ computed from $f^{(m)^{-1}}$ and $p\left(x_{k}^{(n-m)} \mid x_{k}^{(m)}\right)=$ $\delta\left(x_{k}^{(n-m) \leftarrow}-f^{(n-m)}\right)$, a Dirac delta function.

The likelihood function $p\left(y_{k} \mid x_{k}\right)$ is obtained as

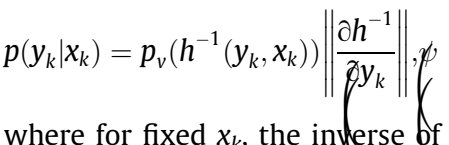

where for fixed $x_{k}$, the inverse of $h\left(x_{k}, v_{k}\right)$ is $h^{-1}$. The denominator $p\left(y_{k}\right)$ is a normalizing constant independent of the states. State estimates are drawn by maximizing or computing the appropriate moments of the conditional pdf. 


\section{Practical approaches to nonlinear estimation}

\subsection{Moving horizon estimation}

Moving horizon estimation aims to construct a conditional joint probability density function for a sequence of discrete state trajectory in a horizon $H$. The joint pdf is maximized subject to constraints on states and inputs to locate the state estimates for the entire horizon

$\max _{\hat{x}_{k-H, k}} p\left(x_{k-H, \ldots, k} \mid y_{k-H, \ldots, k}\right) \cdot \psi$

Using Bayes rule the joint density can be written as

$p\left(x_{k-H, \ldots, k} \mid y_{k-H, \ldots, k}\right)=\frac{p\left(y_{k-H, \ldots, k} \mid x_{k-H, \ldots, k}\right) p\left(x_{k-H, \ldots, k} \mid y_{0, \ldots, k-H-1}\right) \overleftarrow{ }}{p\left(y_{k-H, \ldots, k}\right) \leftarrow} . \psi$

The Markovian nature of the random variables allows us to write

$$
\begin{aligned}
p\left(x_{k-H, \ldots, k} \mid y_{k-H, \ldots, k}\right)= & \frac{1}{p\left(y_{k-H, \ldots, k}\right)} \prod_{j=k-H}^{k} p\left(y_{j} \mid x_{j}\right) \prod_{j=k-H}^{k-1} p\left(x_{j+1} \mid x_{j}\right) \leftarrow \\
& \times f\left(p\left(x_{k-H} \mid x_{k-H-1}\right) p\left(x_{k-H-1} \mid y_{k-H-1}\right) \mathrm{d} x_{k-H-1}, \psi\right.
\end{aligned}
$$

where the integral term summarizes the past information at the beginning of the horizon. For an infinite horizon Eq. (14) contains all the information to draw the estimates. In practice when a finite horizon is moved forward, the conditional density $p\left(x_{k-H-1} \mid y_{k-H-1}\right)$ in Eq. (14) must be updated to $p\left(x_{k-H} \mid y_{k-H}\right)$ for the new horizon using the recursive update in Eq. (8).

The general MHE formulation only requires that the nonlinear functions $f$ and $h$ are nonsingular and continuously differentiable functions of their arguments in order to formulate the joint pdf analytically. With no further assumptions, this nonlinear optimization approach may need global optimization on the objective function generated by the joint pdf.

In order to pose tractable objective functions a few simplifying assumptions are often used that aid in practical implementation of MHE:

- $\leftrightarrow$ Noise processes are additive random variables distributed according to Gaussian pdfs or truncated variants.

- The joint pdf is an exponential function such that the negative logarithm may be minimized.

- $\leftrightarrow$ Past information is summarized by a mean vector and covariance matrix of the state, which is recursively updated by linearized-Gaussian dynamics.

- All variables belong to closed and convex sets.

Under these assumptions, the MHE problem statement is

$$
\begin{aligned}
& \min _{\hat{x}_{k-H, \ldots, k}}\left(x_{k-H}-\mu\right)^{\mathrm{T}} P^{-1}\left(x_{k-H}-\mu\right)+\sum_{j=k-H}^{k-1} w_{j}^{\mathrm{T}} Q^{-1} w_{j} \\
& +\sum_{j=k-H}^{k}\left(v_{j}^{\mathrm{T}} R^{-1} v_{j}, \psi\right.
\end{aligned}
$$

where mean vector $\mu$ and covariance matrix $P$ summarize the past measurements. The first term in the objective function is known as the arrival cost penalizing the errors in the summary of past or prior information. The subsequent summation terms are collectively known as stage costs intended to penalize model and measurement errors, respectively. $Q$ and $R$ are the covariance matrices of process and measurement noise processes, respectively.

The arrival cost is updated by recursive computation of $\mu$ and $P$ using a time-varying linearized state estimator such as the extended Kalman filter or smoother. The problem of accurately summarizing the past information as arrival cost remains an open issue in MHE [17]. The ability to impose meaningful constraints on estimates in the optimization framework is an appealing feature of MHE. The use of a horizon of data, instead of recursive estimation using one measurement at a time, gives the MHE robustness to modeling and parameter errors and unknown disturbances.

\subsection{Cell filter}

The support of the conditional density $p\left(x_{k} \mid y_{k}\right)$ in Eq. (8), is a finite region of state space $\mathbb{X} \subset \mathbb{R}^{n}$, such that constraints on the states restrict the dynamics of the state vector inside $\mathbb{X}$. The space outside the constrained region $\overline{\mathbb{X}}=\mathbb{R}^{n}-\mathbb{X}$, is called a sink cell $z^{0}$. When each state variable is discretized into a collection of indivisible intervals, it forms a state cell space $\mathbb{Z}=\left\{z^{i}: i=0,1, \ldots ; N\right\}$ where $z$ is an n-tuple position identifier of a cell. The cell filter is a piecewise constant approximation of the conditional density supported on the discrete cell space $\mathbb{Z}$.

If the probability associated with a cell $z^{i}$ is denoted by $m_{k}^{i}$ at instance $k$, then the cell probabilities are represented by the probability mass vector (pmv),

$p\left(z_{k}\right)=\left[m_{k}^{0}, m_{k}^{1}, \ldots ; m_{k}^{N}{ }^{\mathrm{T}}\right.$ with $\sum_{i=0}^{N} m_{k}^{i}=1 . \psi$

The probabilistic behavior of the dynamics in cell space can be associated with a stationary Markov chain. The transition probability of the system being in cell $z^{i}$ knowing that the system is currently in cell $z^{j}$ is written as,

$p^{i j}=\operatorname{Prob}\left\{x_{k}\right.$ is in $z^{i} \mid x_{k-1}$ is in $\left.z^{j}\right\}, \psi i, j=0,1, \ldots ; N . \psi$

The elements $p^{i j}$ form an $(N+1) \times(N+1)$ stochastic matrix $\mathbf{P}$, which is the transition probability matrix of the finite state Markov chain. Now we may numerically approximate the integral term in Eq. (8) using the following linear transformation,

$\mathbf{p}\left(z_{k}\right)=\mathbf{P}_{\mathbf{p}}\left(z_{k-1}\right) \cdot \psi$

A piecewise constant approximation of the likelihood function $p\left(y_{k} \mid x_{k}\right)$ is denoted as a likelihood mass vector ( $\left.\operatorname{lmv}\right), \mathbf{1}\left(z_{k}, y_{k}\right)$ supported on the cell space. In case of invertible and differentiable measurement function $h$, the lmv is readily computed by Eq. (11) at cell centers $\bar{z}$ [12]. Otherwise a region of interest in the output

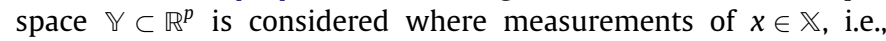
$y \in \mathbb{Y}$ are likely to be obtained. $\mathbb{Y}$ is discretized into a finite set of output cells and $\bar{Y}=\mathbb{R}^{p}-\mathbb{X}$ is the output sink cell $d_{0}$, forming $\mathbb{D}=\left\{d^{i}:-i=0,1, \ldots ; L\right\}$. The likelihood of an output cell $d^{i}$ being the measurement of state cell $z^{j}$ is,

$l^{i j}=\operatorname{Prob}\left\{y_{k}\right.$ is in $d^{i} \mid x_{k}$ is in $\left.z^{j}\right\}, \psi i=0,1, \ldots ; L ;-j=0,1, \ldots ; N . \psi$

The $(L+1) \times(N+1)$ likelihood mass matrix $\mathbf{L}$ contains the elements $l^{i j}$ which is a discretized approximation of the likelihood function in Eq. (11). Given the output $y_{k}$, which falls in the output cell $d_{k}^{i}$ the $\operatorname{lmv} \mathbf{l}\left(z_{k}, d_{k}^{i}\right)$ is presented by the $i$ th row in $\mathbf{L}$.

The cell filter is a recursive computation of the posterior probability mass vector as shown below,

$\mathbf{p}\left(z_{k} \mid y_{k}\right)=\frac{\mathbf{l}\left(z_{k}, d_{k}^{i}\right) \odot \mathbf{P p}\left(z_{k-1} \mid d_{k-1}^{j}\right) \leftarrow}{\left.\sum\left(z_{k}, d_{k}^{i}\right) \odot \mathbf{P p}\left(z_{k-1} \mid d_{k-1}^{j}\right)\right)}, \psi$

where $\odot$ is the Haddamard product. Optimal estimates can be drawn as expectation of any real valued function $\phi(x)$, with a dot product,

$\mathbf{E}\{\phi(x)\} \approx \phi(\overline{\mathbf{z}})^{T} \cdot \mathbf{p}\left(z_{k} \mid y_{k}\right), \psi$ 
where $\phi(\overline{\mathbf{z}})$ is a vector of function values at cell centers. A maximum a posteriori (MAP) estimate can be determined by maximizing the vector $\mathbf{p}\left(z_{k} \mid y_{k}\right)$,

$z_{k}^{\mathrm{MAP}}=\arg \max \mathbf{p}\left(z_{k} \mid y_{k}\right), \psi$

and the MAP estimate $\hat{x}_{k}^{\text {MAP }}$, is taken as the center point of the cell.

The elements of $\mathbf{P}$ are computed using Monte Carlo integration known as generalized cell mapping (GCM) [18]. In Eq. (17) the transition probability is,

$p^{i j} \quad \frac{s^{i}}{s^{j}}, \psi$

where $s^{i}$ are the number of uniformly sampled initial conditions in a cell $z^{j}$, and $s^{i}$ are the number of end points of trajectories culminating in the image cell $z^{i}$. The mapping is performed by implementing the process model in Eq. (1) forced by a constant input $u$ and samples drawn from $p_{w}(w)$. Similarly, the elements of $L$ are obtained by a cell mapping between state and output spaces. They are precomputed from simulated measurements of samples in state cell $z^{j}$ as follows

$l^{i j} \quad \frac{r^{i}}{s^{j}}, \psi$

where $r^{i}$ are the number of measurements located in output cell $d^{i}$. The cell mapping only requires that $f$ and $h$ are computable, hence non-singularity and differentiability are not relevant. This may be a useful feature for estimation of hybrid systems with mixed continuous and discrete states.

Since the argument $u$ is generally continuous in $\mathbb{U}$, an infinite number of transition probability matrices are possible. Noting that the precision of the control input is limited to the precision of an actuator input, it is discretized into $M$ values in $\mathbb{U}$ to define a control cell space $\mathbb{V}=\left\{v^{i}: i=1, \ldots, M\right\}$. For instance, Fig. 1 illustrates GCM on a slice of state space corresponding to a fixed input. Mappings of four samples from $z^{19}$ indicate that $p\left(z^{2} \mid z^{19}\right)=0.25$, $p\left(z^{7} \mid z^{19}\right)=0.5$ and $p\left(z^{12} \mid z^{19}\right)=0.25$. A family of $M$ transition probability matrices $\mathbf{P}\left(v^{i}\right)$ define a finite state stationary Markov chain to describe the probabilistic behavior of the system in state and input spaces.

The online algorithm for state estimation in a closed loop setting is summarized by the following steps:

(1) Initialize cell filter with $\mathbf{p}\left(z_{k-1} \mid y_{k-1}\right)$ at $k$.

(2) Obtain $u_{k}$ from controller. Locate control cell $v^{i}$ containing $u_{k}$. Load $\mathbf{P}\left(v^{i}\right)$ into memory.

(3) Predict a priori pmv with linear transformation in Eq. (18).

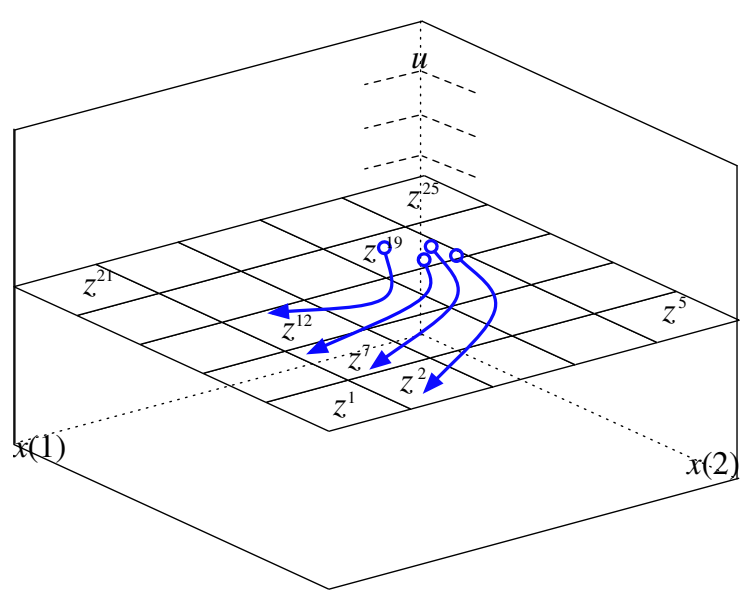

Fig. 1. Generalized cell mapping in state and input cell space.
(4) Obtain measurement $y_{k}$. Compute $\operatorname{lmv} \mathbf{l}\left(z_{k}, y_{k}\right)$ with Eq. (11) or lookup ith row $\mathbf{l}\left(z_{k}, d_{k}^{i}\right)$ from matrix $\mathbf{L}$.

(5) Compute a posteriori pmv $\mathbf{p}\left(z_{k} \mid y_{k}\right)$ with Eq. (20).

(6) Compute expectation in Eq. (21) or MAP estimate in Eq. (22) for controller.

(7) Return to step (2) for $k+1$.

Much of the online computational cost incurred is due to a large sparse matrix-vector multiplication in step (3), which can be small compared to a nonlinear program solved online. The benefits of the cell filter over MHE include:

- Commonly used simplifying assumptions are not necessary. It may be regarded as a numerical approximation of the general MHE solution in a horizon of one, without requiring nonlinear optimization.

- $\leftrightarrow$ Provides mean or mode estimates, error statistics and confidence intervals.

- Recursion is on the entire conditional pmv, hence propagation errors in arrival cost is avoided.

- $\leftarrow$ The off-line computational cost for the Markov chain and likelihood matrices a one time burden, which can alleviate the online computational cost for estimation and control of low dimensional systems.

There are several limitations for practical implementations of the cell filter:

- $\leftrightarrow$ Model accuracy is critical because of recursion based on one sample time.

- If model parameters or inputs change, the cell mapping exercise must be repeated all over again.

- Presence of unconstrained variables in the model make the CF unpractical.

- The curse of dimension limits the CF for low dimensional systems because the number of cells in input and output space, $\left(N^{n} \times m^{m}\right)$ explodes with dimension.

\section{Simulation examples}

\subsection{CSTR}

Consider the following pair of nonlinear ODEs governing a continuously stirred tank reactor (CSTR),

$\frac{\mathrm{d} C_{\mathrm{A}}}{\mathrm{d} t}=\frac{F}{V}\left(C_{\mathrm{A}}^{f}-C_{\mathrm{A}}\right)-k_{1} C_{\mathrm{A}} e^{-E_{1} / R T}, \psi$

$\frac{\mathrm{d} C_{\mathrm{B}}}{\mathrm{d} t}=k_{1} C_{\mathrm{A}} e^{-E_{1} / R T}-k_{2} C_{\mathrm{B}} e^{-E_{2} / R T}-\frac{F}{V} C_{\mathrm{B}}, \psi$

comprising the irreversible reactions $A \rightarrow B \rightarrow C$ [3]. The parameters and operating conditions are tabulated in Table 1 . The state vector of the reactor is $X=\left[C_{A}, C_{B}\right]^{T}$, while the reactor temperature $T$ is the manipulated input. The reactor is simulated by numerically integrating the governing equations from the initial conditions $x_{0}=[1,0]^{\mathrm{T}}$. The state of the reactor is sampled at the interval of $\Delta t=0.05$. It is assumed that measurements of only $C_{\mathrm{B}}$ are available,

Table 1

CSTR parameter set

\begin{tabular}{llll}
\hline Parameter & Value & Parameter & Value \\
\hline$k_{1}$ & $7.2 \times 10^{10} / \mathrm{min}$ & $F$ & $100 \mathrm{~L} / \mathrm{min}$ \\
$k_{2}$ & $5.2 \times 10^{10} / \mathrm{min}$ & $V$ & $100 \mathrm{~L}$ \\
$E_{1} / R$ & $8750 \mathrm{~K}$ & $C_{\mathrm{A}}^{f}$ & $1 \mathrm{~mol} / \mathrm{L}$ \\
$E_{2} / R$ & $9700 \mathrm{~K}$ & $\Delta t$ & $0.05 \mathrm{~min}$ \\
\hline
\end{tabular}


which are corrupted by additive zero mean Gaussian noise of variance $0.1^{2}$.

The following noisy discrete-time model is available to the state estimators,

$x_{k+1}=x_{k}+\int_{k \Delta t}^{(k+1) \Delta t} \frac{\mathrm{d} x}{\mathrm{~d} t} \mathrm{~d} \tau+w_{k}, \psi$

where the process noise vector is $w_{k} \sim N\left(0,0.01^{2} I_{2}\right)$. The prior information about the initial condition is available to the estimators as $\hat{x}_{0}=[1,0]^{\mathrm{T}}$ with the covariance matrix $0.001^{2} I_{2}$.

The moving horizon estimator is formulated in a horizon of 3 data points with the tuning parameters $R=0.1^{2}, Q=0.01^{2} I_{2}$ and $P=0.001^{2} I_{2}$. The constrained nonlinear optimization is performed in MATLAB using fmincon function. The discrete-time process model in Eq. (27) is executed outside MAT-LAB using numerical integration by Adams method with CVODE libraries in $C$ language. The arrival cost is calculated by an extended Kalman filter.

The cell filter is designed in the state space bound by $C_{\mathrm{A}} \in[1,0]$ and $C_{B} \in[1,0]$, which is discretized into $50 \times 50$ state cells. Note that the bounds naturally impose constraints on estimates. The manipulated input $\mathrm{T}$ is considered as piecewise constant over the interval $[370,373.5]$ by defining 36 control cells. Generalized cell mapping is implemented using 400 samples per state cell to compute the thirty six transition probability matrices corresponding to the possible control moves. It requires 36 million computations of the discrete-time process model for one step forward from as many initial conditions. The offline numerical integrations are performed using Adams method with CVODE libraries in C language. The sparsity pattern of a typical low resolution $P$ matrix for constant control is shown in Fig. 2. The transition matrices are imported into MAT$\mathrm{LAB}$ environment for online estimation task.

For regulation purposes, it is desired to maintain the concentration of species $B$ at its maximum yield such that the target is $C_{\mathrm{B}}^{t}=0.654 \mathrm{~mol} / \mathrm{L}$ corresponding to the reactor temperature target $T^{t}=371.4 \mathrm{~K}$. The reactor is regulated by optimally adjusting the temperature $T$ while minimizing the cost function in Eq. (6) with weight matrices $Q_{\mathrm{c}}=\operatorname{diag}(0,400), R_{\mathrm{c}}=2$ and $S_{\mathrm{c}}=0$ inside a prediction and control horizon of $2 \mathrm{~min}$. The constrained nonlinear optimization is performed with fmincon function in MATLAB.

At each sampling time, when the regulator returns the optimized control move $T^{*}$ the input cell closest to it from $\mathbb{T}=\{370,370.1, \ldots, 373.5\}$ is located and the corresponding $\mathbf{P}$ is loaded into memory for estimation. The mismatch between the

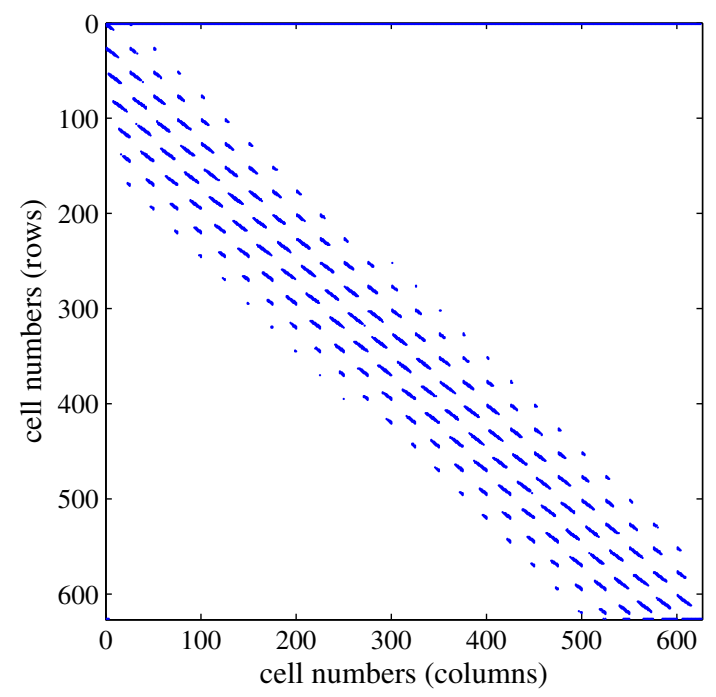

Fig. 2. Sparsity pattern of $\mathbf{P}$ matrix for CSTR example. control cell value of the cell filter and the implemented control move is a random number $T_{\epsilon €}[-0.05,0.05]$. It introduces a model error for the cell filter by perturbing the activation energy in the terms $\exp \left(-\frac{E_{1}}{R\left(T^{*}+T_{\epsilon}\right)}\right)$ and $\exp \left(-\frac{E_{2}}{R\left(T^{*}+T_{\epsilon}\right)}\right)$. Fig. 3 shows the impact of discretization of the control input by showing that $T_{\in}$ perturbs the activation energy between two limits for any given temperature. Simulation results show that the filter is robust to this small discrepancy in $E_{1} / R$ and $E_{2} / R$.

Closed loop simulations were performed using MHE and the cell filter separately as state estimators. Fig. 4 shows the results of closed loop nonlinear model predictive control using the cell filter. A summary of average results for fifty simulation runs are tabulated in Table 2. The offline cell mapping procedure to generate 36 transition probability matrices ran for $27 \mathrm{CPU}$ minutes and stored 29 megabytes of mapping data. By contrast the MHE has no offline computation or storage requirement. The accuracy of the estimators is measured with the following expression,

$\operatorname{MSE}=\frac{1}{K n} \sum_{k=1}^{K}\left(x_{k}-\hat{x}_{k}\right)^{\mathrm{T}}\left(x_{k}-\hat{x}_{k}\right), \psi$

where $K$ is the total number of measurements processed and $n$ is the number of states. On the average the cell filter shows better estimation performance over MHE. The cumulative minimized cost $J_{\text {cumu }}$ is the sum of the costs minimized at each time instance for the length of the simulation. The estimators yielded very close minimums with little variance.

It was observed that the cell filter ran about 400 times faster than MHE. The speed comparison is not relevant because specialized or compiled code for MHE would run faster. In this particular example, since the control horizon is 40 data points wide, the regulation optimization problem has 39 decision variables. In comparison, the estimation horizon for MHE is 3 data points wide with 7 decision variables (initial states and noise variables) for the optimization. Hence regulation optimization required the bulk of the online computations.

\subsection{Agricultural pest control}

Control of agricultural pests using parasitic biological species is an attractive alternative to traditional pesticide based programs. Similar to many predator-prey dynamics, biological pest control also exhibits complicated and often chaotic variations in the pest and parasitic species populations. In this example the population of the larvae of a crop damaging insect is labeled as $l$. The population of a parasitoid, which kills the larvae, is labeled as $p$. The dynamics of the larvae-parasitoid populations are represented by the following set of nonlinear difference equations [19]

$l_{k}=l_{k-1} \mathrm{e}^{c\left(1-\frac{l_{k-1}}{b}\right)} \mathrm{e}^{-a p_{k-1}}+d, \psi$

$p_{k}=l_{k-1}\left(1-\mathrm{e}^{-a p_{k-1}}\right)+u_{k-1} \cdot \psi$

The parameters are $a=1, b=5$ and $c=3$. The initial populations are $l_{0}=3$ and $p_{0}=2$ and a constant supply of new larvae is denoted by $d$. The control input $u$ represents units of parasitoids introduced to exterminate the larvae population. The total cost of the pest control operation $C$, is the sum of the cost of damaged crop due a given larvae population and the cost of the parasitoids introduced.

$C_{K}=c_{l} l_{k}+c_{u} u_{k}, \psi$

where $c_{l}=1.25$ is the crop damage per larva and $c_{u}=1$ is the cost of a unit of parasitoid population. An input disturbance is introduced by doubling the supply of new larvae at $k=25$

$d= \begin{cases}0.1 & 0<k \leqslant 25 \\ 0.2 & k>225 . \psi\end{cases}$ 

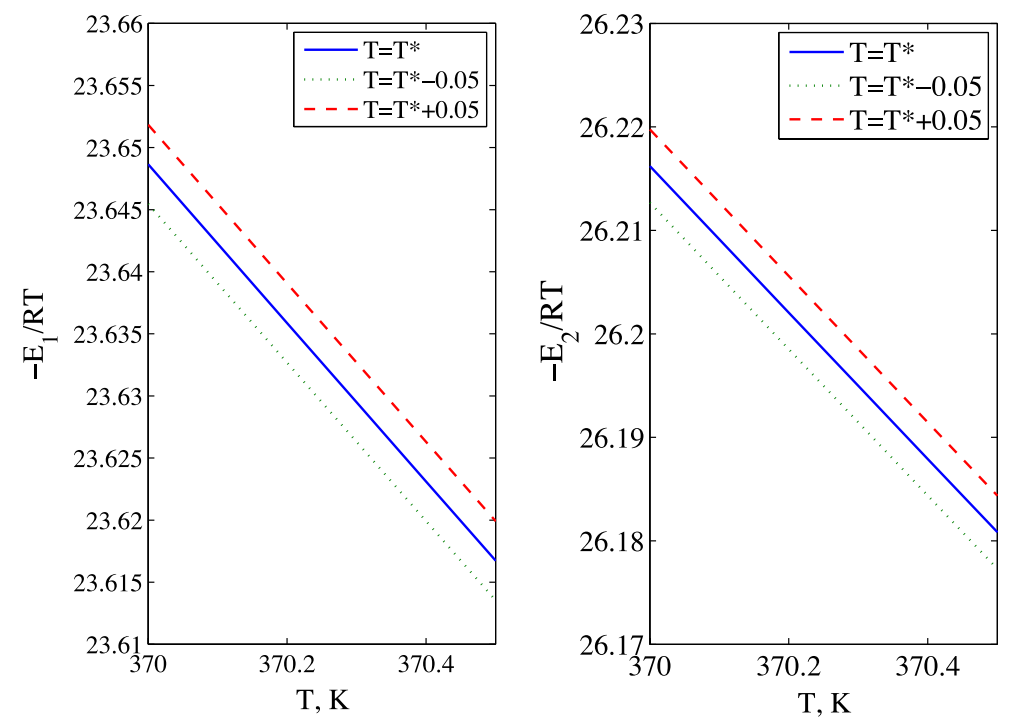

Fig. 3. Influence of $T_{\epsilon} \wp$ n activation energy parameters.
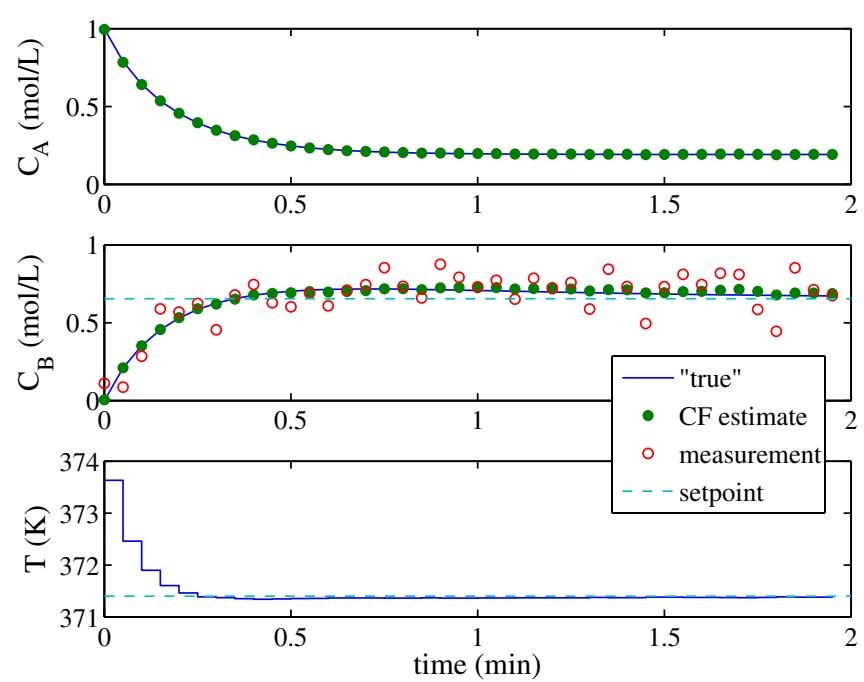

Fig. 4. Closed loop NMPC regulation of CSTR using cell filter.

Table 2

Average results for CSTR example (50 realizations, $3 \mathrm{GHz}$ P4 Linux)

\begin{tabular}{lll}
\hline & MHE & CF \\
\hline Cell mapping CPU time & N/A & $27 \mathrm{~min}$ \\
Memory for storage & N/A & $29 \mathrm{MB}$ \\
Estimation MSE & $15 \times 10^{-5}\left(\sigma=8 \times 10^{-5}\right)$ & $9 \times 10^{-5}\left(\sigma=3 \times 10^{-5}\right)$ \\
Minimized cost $J_{\text {cumu }}$ & $17.4607\left(\sigma=3 \times 10^{-4}\right)$ & $17.4587\left(\sigma=3.5 \times 10^{-4}\right)$ \\
\hline
\end{tabular}

Without the control input $u$ the system exhibits chaotic behavior. Otherwise the system has unstable steady states as well as many quasiperiodic but stable solutions for fixed values of $u$ as shown in dotted lines in Figs. 5 and 6 . The objective is to find a control profile that minimizes the cost of the pest control operation while maintaining stable and even species populations. The performance criterion for model predictive control is

$\Phi=\left(c_{l} l_{k}\right)^{2}+\left(c_{u} u_{k}\right)^{2}, \psi$

with $\Gamma=0$ in Eq. (3)
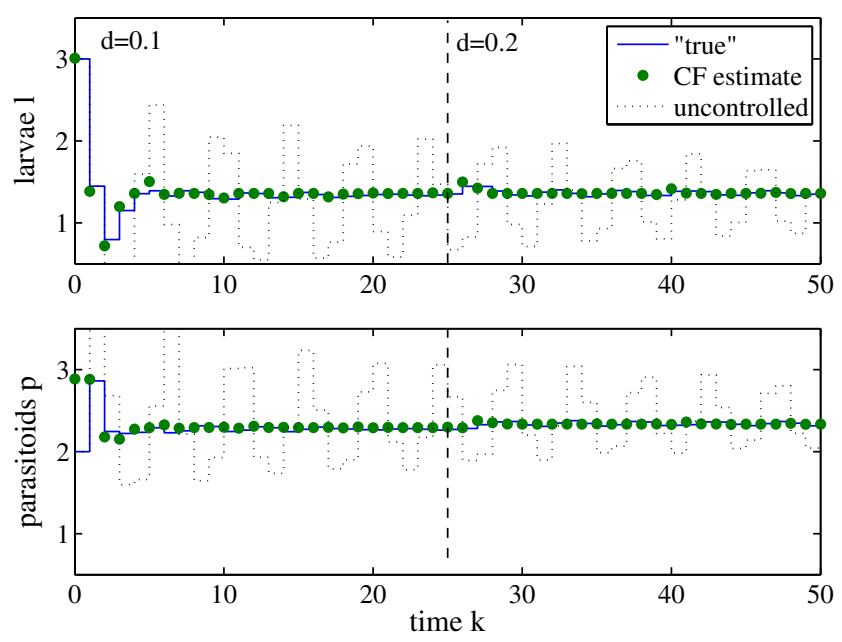

Fig. 5. Closed-loop NMPC control of species populations.
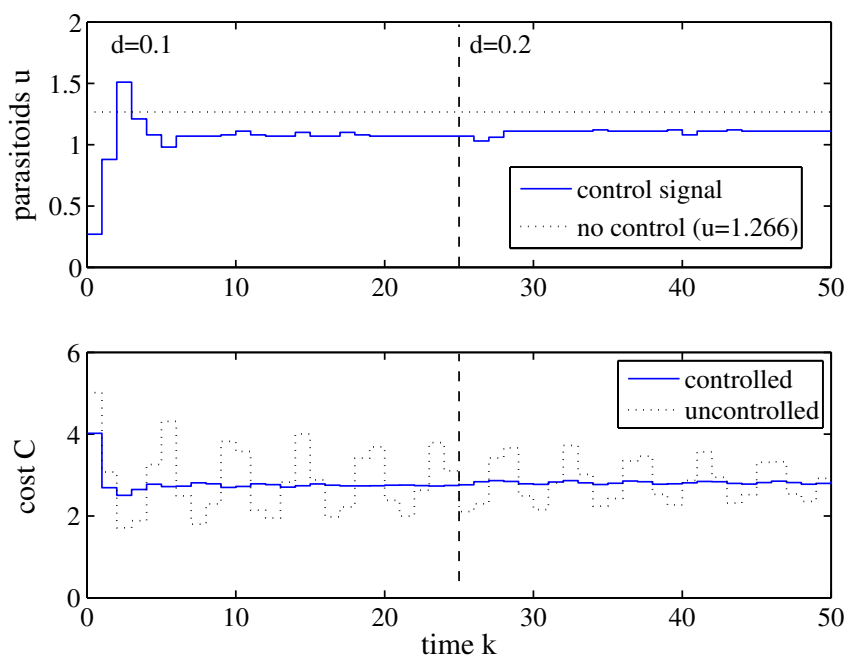

Fig. 6. Optimized control signal and cost. 
Table 3

Average results for pest control example (100 realizations, $3 \mathrm{GHz}$ P4 Linux)

\begin{tabular}{lll}
\hline & MHE & CF \\
\hline Cell mapping CPU time & N/A & 3 min $52 \mathrm{~s}$ \\
Memory for storage & N/A & $9 \mathrm{MB}$ \\
Estimation MSE & $11 \times 40^{-3}\left(\sigma=3 \times 40^{-3}\right)$ & $9.6 \times 40^{-3}\left(\sigma=5 \times 40^{-4}\right)$ \\
Minimized cost $J_{\text {cumu }}$ & $142.83(\sigma=0.02)$ & $142.82(\sigma=0.07)$ \\
\hline
\end{tabular}

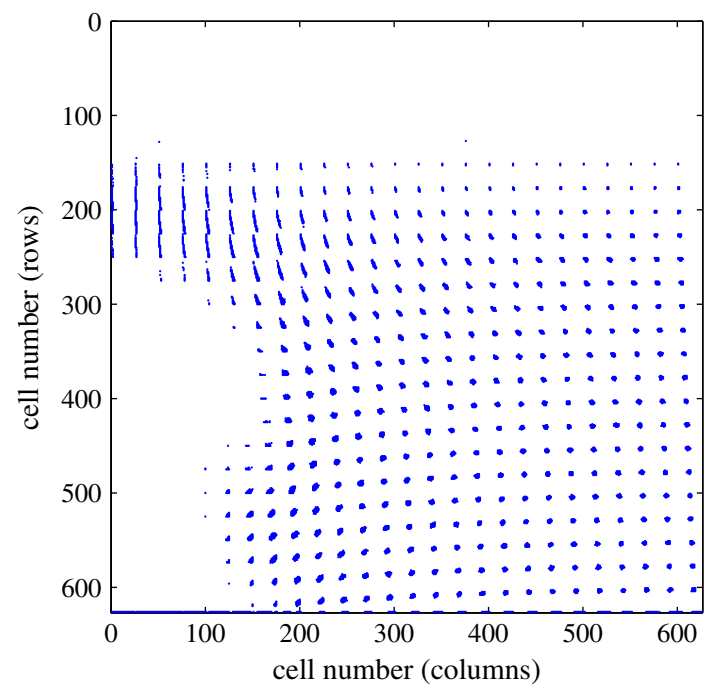

Fig. 7. Sparsity pattern of $\mathbf{P}$ matrix for pest control example.

The state of the process $x_{k}=\left[l_{k}, p_{k}\right]^{\mathrm{T}}$ is not directly accessible. Estimates of the cost of crop damage $y_{k}$, is related to the larvae population by

$y_{k}=\epsilon_{l} l_{k}+v_{k}, \psi$

where $v_{k} \sim N\left(0,0.05^{2}\right)$ represents the errors in the damage estimates. An estimator must be used to reconstruct the state $x_{k}$ from the measurements $y_{k}$ using the noisy model

$x_{k}=f\left(x_{k-1}, d, u_{k-1}\right)+w_{k}$

where $f$ refers to the functions in Eqs. (29) and (30) and $w_{k} \sim N\left(0,0.01^{2} I_{2}\right)$. The initial state of the estimator is poorly known, which is assumed to be $\hat{x}_{0} \sim N\left([2,3]^{\mathrm{T}}, I_{2}\right)$. In addition, since all the variables are related to populations, negative values for states and control are prohibited.

The state space $l, p \in[0,4]$ is discretized into $25 \times 25$ cells and the control variable $u \in[0,2]$ is considered as 201 cells. A fine resolution is necessary for the control cells because the system is sensitive to small changes in input. Considering the two values of the input disturbance in Eq. (32), the cell mapping procedure with 400 samples per cell requires 10.05 million mappings. A total of 402 transition probability matrices are computed in less than 4 min (see Table 3) and stored about 9 MB of mapping data. Fig. 7 shows the sparsity pattern of a typical $\mathbf{P}$, the spread of which reflects a sensitivity of the dynamics to initial conditions.

The results of nonlinear model predictive control based on cell filter estimation are shown in Fig. 5. The control signal profile and the cost of pest control operation are shown in Fig. 6. The populations are quickly stabilized and the disturbance at $k=25$ is also effectively rejected with little disruption.
In this example the control optimization has 4 decision variables and the MHE optimization has 7 decision variables, hence, MHE takes longer than regulation itself, which is not desirable for real-time applications.

\section{Conclusions}

In this paper we proposed to use cell filter for providing state estimates to closed-loop nonlinear model predictive control. For low dimensional systems the cell filter is a practical numerical implementation of a truly recursive Bayesian state estimator for a wide class of nonlinear systems. No simplifying assumptions about the process model or noise processes are necessary, constraints are easily handled and most importantly no realtime optimization is necessary. Simulation studies on nonlinear CSTR and agricultural pest control problems demonstrate comparable performance with MHE. The computational burden of building the Markov chain grows exponentially with the dimensionality of the system, hence, memory and computational burdens currently limit the approach to dimensions of about four or five.

\section{Acknowledgement}

This material is based upon work supported by the National Science Foundation under Grant No. CTS-0522864.

\section{References}

[1] S.J. Qin, T.A. Badgwell, A survey of industrial model predictive control technology, Control Eng. Practice 11 (7) (1993) 733.

[2] R. Findeisen, F. Allgower, Computational delay in nonlinear model predictive control, in: ADCHEM2003, Hong Kong, 2003.

[3] M.J. Tenny, J.B. Rawlings, S.J. Wright, Closed-loop behavior of nonlinear model predictive control, AIChE J. 50 (9) (2004) 2142-2154.

[4] M. Diehl, H.G. Bock, J.P. Schloder, A real time iteration scheme for nonlinear optimization in optimal feedback control, SIAM J. Control Optim. 43 (5) (2005) 1714-11736.

[5] J.D. Hedengren, T.F. Edgar, In situ adaptive tabulation for real-time control, Ind Eng. Chem. Res. 44 (2005) 2716-2724.

[6] J.H. Lee, N.L. Ricker, Extended Kalman filter based nonlinear model predictive control, Ind. Eng. Chem. Res. 33 (1994) 1530-1541.

[7] P.K. Findeisen, Moving horizon estimation of discrete time systems, MS thesis, University of Wisconsin, Madison, WI (1997).

[8] D.G. Robertson, J.H. Lee, J.B. Rawlings, A moving horizon-based approach to least squares estimation, AIChE J. 42 (8) (1996) 2209.

[9] C.V. Rao, J.B. Rawlings, Constrained process monitoring: moving-horizon approach, AIChE J. 48 (1) (2002) 97-109.

[10] W.S. Chen, B.R. Bakshi, P.K. Goel, S. Ungarala, Bayesian estimation via sequential monte carlo sampling: unconstrained nonlinear dynamic systems, Ind. Eng. Chem. Res. 43 (2004) 4012-4025.

[11] J.B. Rawlings, B.R. Bakshi, Particle filtering and moving horizon estimation, Comp. Chem. Eng. 30 (2006) 1529-1541.

[12] S. Ungarala, Z.Z. Chen, K. Li, Bayesian state estimation of nonlinear systems using approximate aggregate Markov chains, Ind. Eng. Chem. Res. 45 (2006) 4208-4221.

[13] L. Lang, W.S. Chen, B.R. Bakshi, P.K. Goel, S. Ungarala, Bayesian estimation via sequential monte carlo sampling - constrained dynamic systems, Automatica 43 (9) (2007) 1615-1622.

[14] S. Ungarala, K. Li, Z. Chen, On constrained Bayesian state estimation using cell filter, Ind. Eng. Chem. Res. (2008) in press.

[15] D. Chmielewski, V. Manousiouthakis, On constrained infinite time linear quadratic optimal control, Syst. Control. Lett. 29 (1996) 121.

[16] A.H. Jazwinski, Stochastic Processes and Filtering Theory, Academic Press, New York, 1970.

[17] E.L. Haseltine, Systems analysis of stochastic and population balance models for chemically reacting systems, Ph.D. thesis, Department of Chemical Engineering, University of Wisconsin-Madison (2005).

[18] C.S. Hsu, Cell-to-cell mapping: a method of global analysis for nonlinear systems, Applied Mathematical Sciences, vol. 64, Springer-Verlag, New York, 1987.

[19] B. Roset, H. Nijmeijer, Observer-based model predictive control, Int. J. Control 77 (17) (2004) 1452-1462. 